\title{
+Spaces: Intelligent Virtual Spaces for eGovernment
}

\begin{abstract}
Intelligent Environments most commonly take a physical form such as homes, offices, hotels, restaurants, shops, that are equipped with advanced networked computer based systems, which enable better or new lifestyles for people. However, Intelligent Environments can also take the form of virtual online spaces such as SecondLife, which can both mimic the real world and provide functionalities which could not be provided in reality, such as advanced simulations and movement. There is the growing trend for people to spend more time in such virtual environments and, to these ends, this work in progress paper reports on a new project, +Spaces which is developing a range of virtual world tools for e-government applications, and presents some of the concepts and technical challenges involved in creating these intelligent virtual spaces for e-government.
\end{abstract}

Keywords: virtual worlds, intelligent spaces, simulation

\section{INTRODUCTION}

There is a great deal of interest in using immersive virtual environments for a range of different applications. Much of this has been stimulated by the success of commercial platforms such as the World of Warcraft for online gaming, and Second Life ${ }^{\circledR}$ for online social networking and e-commerce. These environments have a high level of realism and associated levels of engagement as well as supporting and encouraging social interaction. Davies [1] reported on how virtual worlds can be used to simulate real spaces such as an intelligent campus. He described an iCampus, which allows people to inhabit a virtual environment interacting with each other and with devices in a similar way to a real campus. Within this space there were also simulations of smart spaces which included the University of Essex iSpace and MiRTLE facilities [2].

The + Spaces project (Positiv Spaces - Policy Simulation in Virtual Spaces) is exploring how virtual world technologies can be used to allow government bodies to measure public opinion on a large scale and maximize the value from prospective policy measures by leveraging the power of these communities. +Spaces will be building a range of virtual intelligent environments which range from simple polling applications to more advanced policy simulation spaces.

This paper presents some of the scenarios from + Spaces that illustrate how virtual worlds can be used to create online smart spaces for mass participation of citizens. This is followed by a discussion of some of the technical challenges; and particularly the need for cross-platform independent middleware. We then consider how these spaces can be made more 'intelligent' through the use of integrated reputation and recommendation systems.

\section{VIRTUAL WORLDS FOR E-GOVERNMENT}

Government agencies seek to refine their policy-making processes by assessing the impact of prospective policies on the society prior to their implementation. Traditional tools for this include polls and forums, but these have limited capabilities and do not address many of the issues arising from real life behaviors. +Spaces aims to address this issue by using virtual worlds as testbeds for policy simulation. Contemporary virtual worlds can be viewed as micro-societies with dynamics resembling those of real world societies; the most evolved having virtual economies as well as regulations analogous to a legislative framework. Moreover, virtual worlds are controlled environments in which all parameters of users' reactions and interactions can be tracked. +Spaces will provide governments with the means for testing their legislation in a number of virtual environments as well as data mining tools for tracking the users' reactions. We are developing technologies for aggregating, filtering and analysing this information, as well as recommendation and reputation systems that will increase the relevance to end users and ensure that only reliable sources of data are taken into account. The information derived from these environments will then be represented in an appropriate fashion that will enable government bodies to draw conclusions on the potential outcomes of the policies they propose, taking into account the analogies between virtual world and real life concepts. The +Spaces platform will constitute a powerful tool for testing policies and forecasting their impact and will assist government bodies to include real public opinion in the policy making process. +Spaces also aims to increase participation in this process and extend it to new social communities who are currently not heard.

In the context of +Spaces, the term "Virtual Worlds" is not only restricted to the notion of complex virtualized environments such as SecondLife (which also emulate the socio-economic aspects of real-life through an open-market concept), but we also refer to simple environments that allow users to socialize online and within specific contexts. For example, a forum for education also provides a good space for people to act and socialize in education-related issues (e.g. the Apple Learning Exchange [8]).

The number of people using virtual worlds is increasing at a rate of $15 \%$ every month [3] and does not show signs of stopping or slowing down anytime soon. Indeed, to the contrary, Gartner analysts predict that by the end of 2011, 80 percent of active Internet users will be members of a virtual world, raising the virtual world population to 50 million.

The most evolved virtual worlds have rules and regulations analogous to a legislative framework. Moreover, in virtual worlds such as massively multi player online role-playing games (MMORPGs) and life simulation games, virtual economies exist with their own currency and rules defining the 
possession and persistence of property. Spontaneous effects including mass-protests, crime, harassment and even a plague, with consequent population migrations, have been witnessed in these environments. In each of these extreme examples (taking into consideration the current state-of-the-art), people participating in those virtual worlds tend to extend their personality through their avatars. This is made possible through the interaction with the world. The more a person gets involved in a virtual world, the more the avatar can become an extension of their actual character [4].

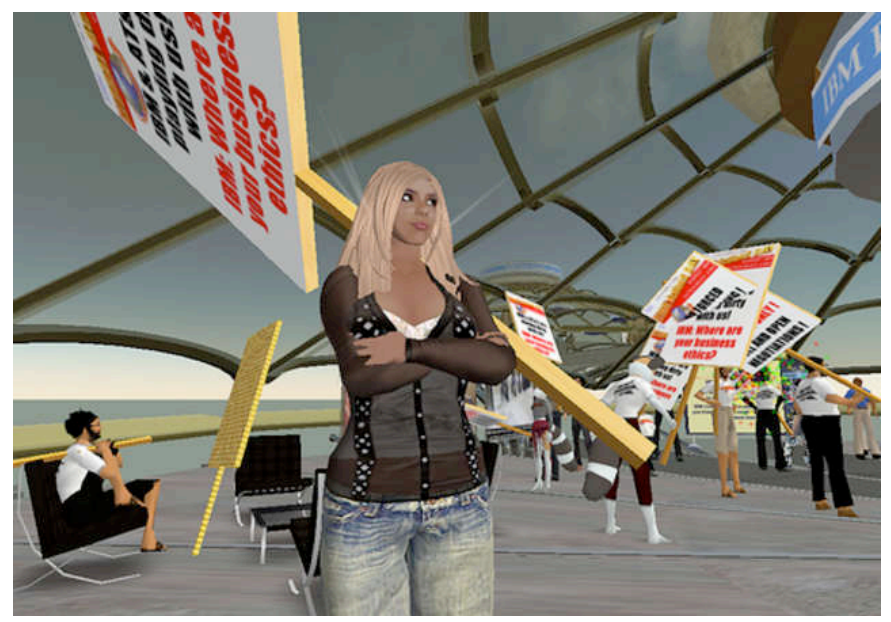

Figure 1. Virtual demonstrations

The existence of these conditions creates an economic system with properties similar to those seen in contemporary economies. Several aspects of economic theory could therefore be used to study these virtual worlds and significant research has been conducted towards that direction of relating virtual world economics to the economics of the real world [5, $6,7]$.

Importantly, people participating in virtual worlds originate from different socioeconomic backgrounds with various beliefs and values. Moreover, they interact with each other in ways similar to what we see in real world. In addition, the relative liberty and anonymity between users, provided through the use of avatars, can reduce the power of peer pressure to conform to societal or cultural norms and stereotypes. For example, in 2007, Italian workers of an international organization went on strike. About 1850 workers expressed their strike related activities across the organization's various sites in Second Life. Due to the novelty, this was picked up by global media and possibly was a factor in the quick resolution of the strike. This is illustrated in Figure 1.

\section{VIRTUAL WORLD SCENARIOS}

+Spaces is creating a number of policy making applications and specifically will be exploring scenarios based on allowing large numbers of citizens to be able to take part in polling, debate and policy simulation within virtual worlds. These scenarios have been selected because of the incremental difficulty that they present in their implementation, their relation to the overall vision and the demand to support them from the +Spaces end user, which is the Hellenic Parliament in Greece. In detail the + Spaces scenarios are:

\section{A. Polling}

A polling application is being created as the simplest policy making application to run in + Spaces. The purpose is to investigate policy issues that only require simple feedback from citizens, such as "what is the society percentage that agrees to a new bill". From a virtual world point of view this scenario can be implemented as a simple forum where people provide feedback through their avatars. However virtual worlds give us the opportunity to conduct polls differently, such as by interviewing people walking by, or by installing two doors and letting people walk through the one they want to vote for, or by asking people to stand on different sides of a room. From a technical perspective, the platform will need to manage each user's privacy across a range of virtual world environments including Second Life [9], OpenSim [10], and Project Wonderland [11].

\section{B. Debating}

This scenario is more focused on seeking qualitative feedback from members of the society. A key factor will be the ability to host public debates with government people who can attend different virtual worlds at the same time. This will allow users within these different worlds to experience the sense of participating in a meeting, hearing opinions, and even expressing their opinions. This also raises additional challenges such as being able to augment the virtual world with reputation systems to help qualify each individual's response in the debate.

This scenario is illustrated by the work of Drew Harry [12], which has explored novel ways of using virtual worlds to support meetings, and includes elements of both polling and debates. An example of when an avatar takes a stance on an issue is shown in figure 2. In this example a cylinder grows up around the avatar, signifying how long it has held the same position. If the avatar moves (representing a change of opinion) the cylinder remains, slowly diminishing. In this way a person's previous opinions will still be apparent, representing the effect that the opinion continues to have on the conversation. A set of cylinders above an avatar's head tracks its motion during a meeting. The path is a record of one person's changing positions on an issue. Various colouring strategies can be used to convey additional information, such as using the colours to match those on the floor, as a way of revealing the same spectrum of agreement. 


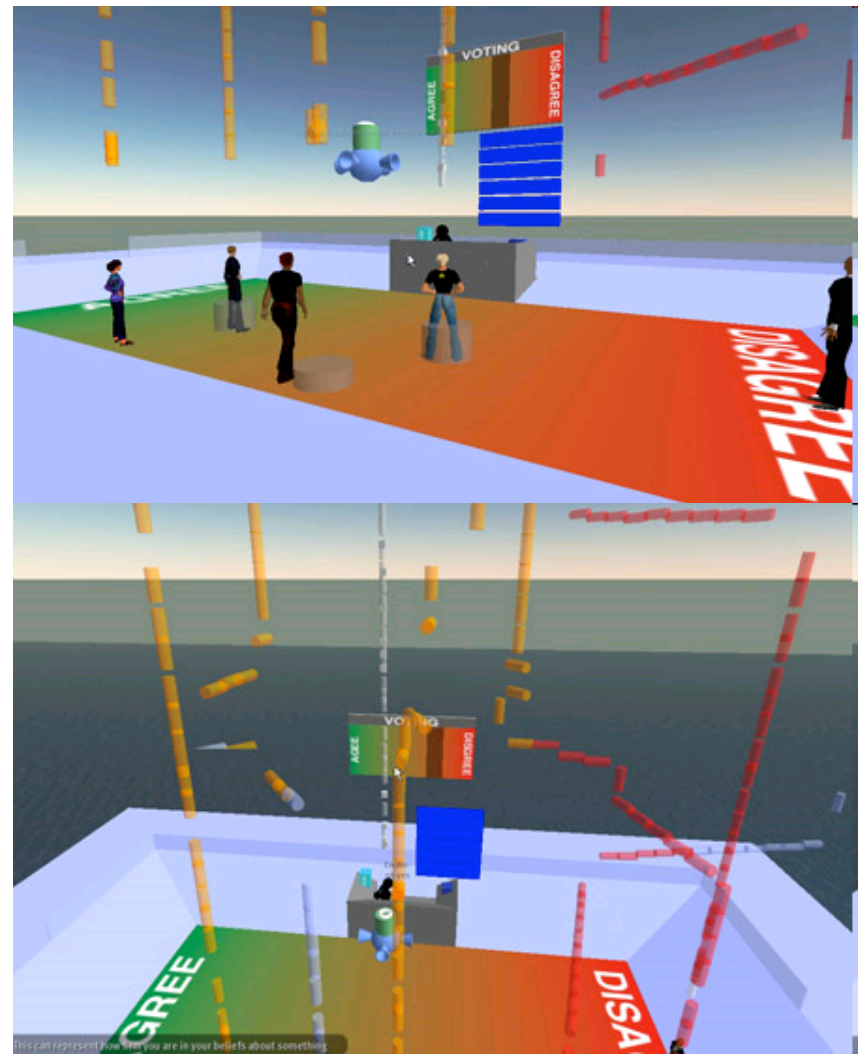

Figure 2. Debating and polling in virtual worlds

\section{Policy and Decisions Simulation}

This scenario allows governmental agencies to use the +Spaces tools and technologies to perform societal simulations, integrating all possible variables, parameters, and scenarios necessary to forecast potential outcomes and impacts of proposed policy measures. +Spaces builds on these methodologies by using an environment that assimilates society's aspects, thus capturing a "snapshot" of the behaviour of the (real world) society if the governmental policies were to be deployed there.

\section{Scenario}

Here is a scenario that illustrates each of these application types:

The Ministry of Justice decided to amend the recently passed law entitled 'Contract of Voluntary Co-habitation', which regulates the matter of the formal voluntary co-habitation of two persons. They start to investigate the issue by initiating ePolls related to the legislation under debate. An indicative example could be:

I believe that the Contract of Voluntary Co-habitation'
should be amended
- I agree
- I disagree
- I am not interested

Additionally, structured debates are formulated in order to involve the virtual citizens either to agree or disagree or provide alternative suggestions or comments.

The government collects all the relevant feedback in order to proceed with further analysis and the collection of the main facts derived from the debate process. The results of this debate will then be considered and will be taken into account in the proposed bill amendment.

Finally, they would like to investigate the impact of the proposed policy. All relevant facts deriving from the debate phase are then simulated and evaluated within the virtual communities in order to analyze the reaction of the community members. If for example, the unmarried couples start adopting children, couples of the same sex are also included so that they have the right to form a union under the scheme that the law regulates, etc.

\section{TECHNICAL CHALLENGES}

One of the key objectives of + Spaces is to implement these application scenarios across a range of different virtual world platforms. In an attempt to promote virtual world interoperability as well as encourage third-parties to run servers that connect to their platform, Linden Lab, the owner of Second Life and the virtual world platform Second Life Grid, have announced their intention to develop open and wellspecified standards for virtual world interfaces and data representation. The architecture working-group has already submitted a first draft of their proposed standards, the Open Grid Protocol to the Internet Engineering Task Force (IETF). The main innovation introduced in this draft protocol is the Linden Lab Structured Data (LLSD), a language-neutral facility for maintaining and transporting structured data which includes an abstract type system, an interface description language and several serialization schemes. In Europe, the standardization work is being focused on MPEG-V10, and is led by Philips Research. The intended standard for information exchange with virtual worlds will consist of at least three parts: a) Architecture, b) Interfaces between virtual worlds and c) Interfaces between virtual worlds and the physical world.

At the moment, however, these standards are at a draft status, and it is yet to be determined whether other virtual world providers will agree to conform, leading to a widely accepted protocol.

In order to accommodate the requirements for interoperability with numerous and heterogeneous virtual world platforms, while at the same time ensuring scalability and robustness, +Spaces shifts the focus from pure interoperability to the support for cross-platform application development and deployment. The idea is to abstract the virtual world functionality to a basic set of services that are exposed in a standardized way (thus solving interoperability on a different level), creating a so-called middleware. By hosting the services outside of the Virtual World platforms together with the appropriate lifecycle management mechanisms enables their combination into larger application workflows. Thus, +Spaces 
delivers both a platform and a software as a service. This is a typical paradigm of a cloud computing approach built on top of a Service Oriented Architecture (SOA), designed to support interoperable machine-to-machine interaction over a network [13].

A SOA that is based on loosely coupled distribute services ensures location independence from the underlying systems. Loose coupling also ensures flexibility and fault tolerance, enabling the creation of a self-healing infrastructure that reduces management costs.

Another major issue in our approach is to achieve exchangeability among the data representations used in different virtual worlds. The main challenge in establishing virtual world interoperability at that level is to identify the different levels of abstraction present in each virtual world and define a generalised data abstraction layer. This can then be used to describe all virtual world entities using concepts that make sense in the various virtual worlds. They will then be transformed to the + Spaces generic data representation in order to be appropriately processed and analysed.

The high level architecture for + Spaces is illustrated in figure 3. The +Spaces API will act as the middleware layer between the underlying virtual world platforms (OpenSim, Wonderland, SecondLife), and the eGovernment application layer, which will allow the policy leaders to deploy and manage the polling, debating and simulation activities on the platform. In addition the +Spaces API will provide common infrastructure services such as authentication and identity management, and links to reputation and recommendation systems, which can be used to further analyse the data derived from the virtual worlds.
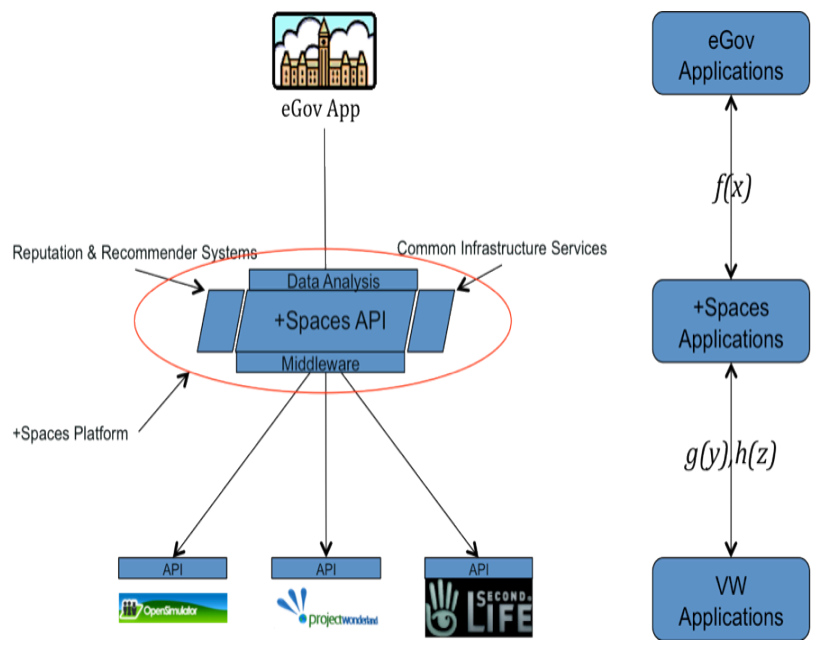

Figure 3. +Spaces architecture

\section{CREating Intelligent Spaces}

In addition to exploring novel ways of using virtual worlds to support a range of e-Government applications (polling, debating and simulation), +Spaces will also use data mining methods to augment the in-world experience, and allow policy makers to extract additional information from the virtual world sessions. This will be achieved through the use of recommendation and reputation systems that integrate with the + Spaces middleware layer.

Recommendation systems are commonplace in a variety of web applications such as social networks, and commercial ecommerce sites. Various methods are used for creating recommendations including content-based, knowledge-based, demographic, and hybrid recommendations, which combine two or more of the other methods (see [14] for more detail). Collaborative filtering [15], which has become the most popular technique for making recommendations on websites, is based on user preference similarity. In recent years, as social networks sites proliferate, more research has been conducted on leveraging the user's explicit social network. Instead of basing recommendations on similar users, recommendations are based on people the user is familiar with (see [16, 17, 18, 19 and 20]). Social network information can therefore be very useful for enhancing recommendation systems. Combined with more traditional content-based techniques, the user can be recommended with items related to content of interest and that are related to the user's network [21]. The richer the social network, the better recommendations are likely to be. Rich aggregated social information can also be used to enhance recommendation systems that suggest items of different kinds to the user.

In the virtual world context, recommendation systems may recommend interesting people to meet $[20,22]$, places to visit, or objects to consume. In the framework of +Spaces, recommendations will assist by attracting people to different polls and debates that may be of interest to them, based on their friends' behavior; they can make the virtual world "stickier" (more attractive to users), and help with the diffusion of + Spaces applications among users [23]. Filtering polls and debates in order to match each user's special interests will increase the possibility of enticing them into participating and will assist towards creating a critical mass.

According to [24], reputation systems represent a significant trend in decision support for internet mediated service provision and play a major role in online auction and shopping websites such as e-bay where a user's trustworthiness and credibility are of primary concern. Reputation systems use various methods for calculating reputation. These methods include simple summation or averaging of ratings, Bayesian systems, discrete trust models, belief models, fuzzy models, and flow models. Virtual worlds allow the creation of efficient systems for collecting information and for deriving measures of reputation, rather than requiring explicit ratings. For example, popularity of users and positive responses to users in debates could contribute to the users' reputation. Reputation has already been employed in several virtual worlds, and in some cases it was not well accepted by users and even removed (e.g., from Second Life). +Spaces is investigating a reputation system that is based on a users social network, and 
seeks to allow users to manage their reputations based on their preferences and social network as well as their current context.

For + Spaces the reputation information will be useful for ranking and analysing the user behaviour. Although in general the avatar's actions and behaviour are indicative of the user's opinion and beliefs, it is not uncommon to come across users who hide behind their virtual identities in order to act provocatively and disrupt serious discussions. Such behaviour could not only diminish the e-Government application's credibility and discourage potential participants but also undermine the value of the results. In order to alleviate those risks, +Spaces will take advantage of the user's history and the social network information gathered, and develop a socialnetwork based reputation system. This system will be used to assess each user's credibility and trustworthiness and decide on whether their opinion can bring any true value to the policy making process.

\section{CONCLUSION}

Technologically supported environments can take both physical and virtual forms. This paper addresses the latter and has presented work in progress on the +Spaces project - an intelligent virtual space for eGovernment. The work is currently at a "conceptual stage" and as part of our work, this paper presents the underlying models and principles +Spaces is adopting. We have described how we intend to develop a middleware API layer that will integrate into a number of backend virtual worlds. A range of different policy making applications will be deployed in these worlds, which can be used to elicit feedback from potentially large numbers of users/citizens on complex government issues. Ultimately the aim is to be able to simulate these policies within these virtual spaces and extract the rich behavioral data for further analysis using a range of data-mining techniques. This work is pioneering the use of virtual environments as a policy deliberation tool, and as such will address many uncertain and challenging issues ranging from technology through to sociology, all of which will need careful evaluation. It is anticipated that these techniques will allow us to examine these user behaviors in greater detail, and also encourage rich forums for debate and government/citizen engagement. Finally, being a work-in-progress paper the project will be developed over a number of years and we look forward to reporting our progress in subsequent conferences.

\section{ACKNOWLEDGMENT}

+Spaces is funded by the EU Seventh Framework Programme, theme ICT-2009.7.3 ICT for Governance and Policy Modelling under Contract No. 248726.

\section{REFERENCES}

[1] M. Davies, V Callaghan and M Gardner, "Towards a Mixed Reality Intelligent Campus" in Proceedings of the $4^{\text {th }}$ International Conference on Intelligent Environments, IE04, 2008.

[2] M. Gardner, J. Scott and B. Horan, "Reflections on the use of Project Wonderland as a mixed-reality environment for teaching and learning", ReLIVE 08 conference, Open University, UK, 2008.

[3] Gartner Symposium/ITxpo 2007 Emerging Trends, Press Release

[4] E Castronova, "Synthetic Worlds: The Business and Culture of Online Games", University of Chicago Press (2005). ISBN 0-226-09626-2

[5] E Castronova, "On Virtual Economies", (July 2002). CESifo Working Paper Series No. 752.

[6] C. R.Ondrejka, "Living on the Edge: Digital Worlds Which Embrace the Real World", June 5, 2004.

[7] I. MacInnes, "The Implications of Property Rights in Virtual World Business Models". Americas Conference of Information Systems (AMCIS 2004), New York, NY

[8] Apple Learning Interchange http://edcommunity.apple.com/ali/

[9] SecondLife, http://www.secondlife.com

[10] OpenSim, http://www.opensimulator.org

[11] Wonderland, http://www.projectwonderland.com

[12] D Harry, J Donath. "Information Spaces - Building Meeting Rooms in Virtual Environments" (Works in Progress) In Proceedings of the SIGCHI conference on Human Factors in Computing Systems (CHI 2008) (Florence, Italy).

[13] Web Services Glossary, http://www.w3.org/TR/ws-gloss/

[14] R. Burke, "Hybrid Recommender Systems: Survey and Experiments". User Modeling and User- Adapted Interaction 12, 4 (2002), 331-370.

[15] J. L. Herlocker, J. A. Konstan, and J.Riedl, "Explaining collaborative filtering recommendations". Proc. CSCW'00, (2000), 241-250

[16] R. Sinha, and K. Swearingen, "Comparing Recommendations made by Online Systems and Friends". Proc. DELOS-NSF Workshop on Personalization and Recommender Systems in Digital Libraries, (2001).

[17] P. Bonhard, and M. A. Sasse, "Knowing me, knowing you -- Using profiles and social networking to improve recommender systems". BT Technology Journal 24, 3 (Jul. 2006), 84-98.

[18] E. Spertus, M. Sahami, and O. Buyukkokten, "Evaluating similarity measures: a large-scale study in the Orkut social network". Proc. SIGKDD'05. (2005), 678-684.

[19] W. Geyer, C. Dugan, D. Millen, M. Muller, and J. Freyne, "Recommending Topics for Self- Descriptions in Online User Profiles". Proc. RecSys'08, (2008), 59-66.

[20] I. Guy, I. Ronen, E. Wilcox. "Do You Know? Recommending People to Invite into Your Social Network". Proc. IUI'09, (2009), 77-86.

[21] I. Guy, N. Zwerdling, D. Carmel, I. Ronen, E. Uziel, S. Yogev, S. OfekKoifman, "Personalized recommendation of social software items based on social relations". Proc. RecSys'09, (2009), 53-60

[22] J. Chen, W. Geyer, C. Dugan, M. J. Muller, I. Guy, "Make new friends, but keep the old: recommending people on social networking sites". Proc. CHI'09, (2009), 201-210

[23] J. Freyne, M. Jacovi, I. Guy, W. Geyer, "Increasing engagement through early recommender intervention”. Proc. RecSys'09, (2009), 85-92

[24] A. Jsang, R. Ismail, and C. Boyd, "A survey of trust and reputation systems for online service provision," in Decision Support Systems, 2005 . 